\title{
Correction to: Pricing two-asset alternating barrier options with icicles and their variations
}

\author{
Hangsuck Lee ${ }^{1} \cdot{\text { Eunchae } \mathrm{Kim}^{2} \cdot \text { Seongjoo Song }}^{3}$ iD
}

Published online: 14 February 2020

(c) Korean Statistical Society 2020

\section{Correction to: Journal of the Korean Statistical Society https://doi.org/10.1007/s42952-019-00039-3}

The original version of this article unfortunately contained a mistake.

The correct Eq. (11) should be:

$$
\operatorname{Pr}(X(t) \leq x, M(t) \leq m)=\Phi\left(\frac{x-\mu t}{\sigma \sqrt{t}}\right)-e^{\frac{2 \mu}{\sigma^{2}} m} \Phi\left(\frac{x-2 m-\mu t}{\sigma \sqrt{t}}\right)
$$

The original article can be found online at https://doi.org/10.1007/s42952-019-00039-3.

Seongjoo Song

sjsong@korea.ac.kr

1 Department of Actuarial Science/Mathematics, Sungkyunkwan University, Seoul, Korea

2 Department of Risk Management and Insurance, Robinson College of Business, Georgia State University, Atlanta, USA

3 Department of Statistics, Korea University, Seoul, Korea 